\title{
On the Solution of NP-hard Linear Complementarity Problems
}

\author{
Joaquim J. Júdice* \\ Departamento de Matemática, Universidade de Coimbra, Coimbra, Portugal \\ e-mail: judice@dragao.it.uc.pt \\ Ana M. Faustino and Isabel Martins Ribeiro** \\ Faculdade de Engenharia, Universidade do Porto. Porto, Portugal \\ e-4nail: afauståfe.up.pt iribeiro@fe.up.pt
}

\begin{abstract}
In this paper two enumerative algorithms for the Linear Complementarity Problems $(L C P)$ are discussed. These procedures exploit the equivalence of the $L C P$ into a nonconvex quadratic and a bilinear programs. It is shown that these algorithms are efficient for processing NP-hard LCPS associated with reformulations of the Knapsack problem and should be recommended to solve difficult LCPs.
\end{abstract}

Key Words: mathematical programming, complementarity, global optimization, enumerative algorithms.

AMS subject classification: $90 \mathrm{C} 33,65 \mathrm{~K} 10$.

\section{Introduction}

The Linear Complementarity Problem $(L C P)$ consists of finding vectors $z \in \mathbb{R}^{n}$ and $w \in \mathbb{R}^{n}$ such that

$$
\begin{aligned}
& w=q+M z \\
& z \geq 0, \quad w \geq 0 \\
& z^{T} w=0
\end{aligned}
$$

for a given matrix $M \in \mathbb{R}^{n \times n}$ and a vector $q \in \mathbb{R}^{n}$. This problem has originally appeared in the sixties for the solution of bimatrix games and convex quadratic programs. Since then, it has received an increasing interest,

\footnotetext{
* Support for this author was provided by Instituto de Telecomunicações and by FCT under grant POCTI/35059/MAT/2000

** Support for this author was provided by PRODEP under grant 4/5.3/PRODEP $/ 00$

Manuscript received: September 2001. Final version accepted: June 2002.
} 